\section{Nasal NO, high-speed video microscopy, electron microscopy, and genetics: a primary ciliary dyskinesia puzzle to complete}

doi: 10.1038/pr.2014.79

To the Editor: In the review of Horani, Brody, and Ferkol (1) the advances in the genetics of primary ciliary dyskinesia (PCD) are well described.

We agree with the authors that there has been an explosion in the knowledge related to cilia, and that electron microscopy has been part of the gold standard for diagnosis in PCD in the last years. However, in the 2009 European Respiratory Society consensus statement (2) on diagnostic and treatment approaches in children in both the ciliary beat pattern and frequency analysis using a video recording system and electron microscopy analysis are strongly recommended.

The authors state that high-speed video microscopy is available only at a limited number of research centers and requires sophisticated software and expertise in analysis and should be related to research only. On the contrary, several authors (3-6) in Europe recommend the use of ciliary beat pattern and frequency analysis by high-speed video microscopy as a necessary first step in the diagnosis of PCD. High-speed video microscopy is an essential piece of the puzzle that leads to the definitive diagnosis, as well as the clinical picture, nasal NO measurements, and ultrastructural electron microscopy analysis.

The required equipment varies in specificity and costs, but normal light microscopy with a video recording system that can register at least $25-30$ frames per second is able to calculate frequency and evaluate movement patterns at slowmotion. Obviously training in centers that routinely use this technique is useful to standardizes this technique and obtain comparable results. The sole visualization of absent or reduced beating in repeated samples is indicative for PCD, and can in poor resource countries with no electron microscopy availability lead to a diagnosis when the clinical picture is suggestive.

Moreover, we believe that the biggest advance in PCD diagnostics is not nasal NO measurements as the authors state in their review but the genetics of PCD.

Genetic analysis is useful to identify new atypical phenotypes, although available at the moment only in a limited number of research centers.

Disclosure: No potential or perceived conflict of interests to disclose.

Deborah Snijders', Ilaria Bertozzi' and Angelo Barbato'

${ }^{1}$ Department of Women's and Children's Health, Endoscopic Unit, University of Padua, Padua, Italy.

Correspondence: Deborah Snijders (olanda76@gmail.com)

\section{REFERENCES}

1. Horani A, Brody SL, Ferkol TW. Picking up speed: advances in the genetics of primary ciliary dyskinesia. Pediatr Res 2014;75:158-64.

2. Barbato A, Frischer T, Kuehni CE, et al. Primary ciliary dyskinesia: a consensus statement on diagnostic and treatment approaches in children. Eur Respir J 2009;34:1264-76.

3. Smith CM, Djakow J, Free RC, et al. ciliaFA: a research tool for automated, high-throughput measurement of ciliary beat frequency using freely available software. Cilia 2012;1:14.

4. Armengot M, Milara J, Mata M, Carda C, Cortijo J. Cilia motility and structure in primary and secondary ciliary dyskinesia. Am J Rhinol Allergy 2010;24:175-80.

5. Chilvers MA, Rutman A, O'Callaghan C. Ciliary beat pattern is associated with specific ultrastructural defects in primary ciliary dyskinesia. J Allergy Clin Immunol 2003;112:518-24.

6. Papon JF, Bassinet L, Cariou-Patron G, et al. Quantitative analysis of ciliary beating in primary ciliary dyskinesia: a pilot study. Orphanet J Rare Dis 2012;7:78 